\title{
Von der Doppelgleisigkeit der Verwaltung zur mittelbaren Bundesverwaltung - ein Überblick
}

\section{From 'Double Track' Administration to Indirect Federal Administration - an Overview}

This paper describes the changes in the Austrian regional administration from 1918 to 1925, distinguishing four periods (before 1918, 1918-1920, 1920-1925, since 1925). Several problems were discussed during all these periods: The term 'double track' administration was criticised (as manipulatory), and the desired 'democratisation' of the administration stood against the idea of its (judicial) responsibility to parliament. Another problem was the uniformity of administration, which seemed difficult to secure. Due to the large number of public servants within the electorate, the future role of the public service was discussed controversially. Overall, the 1925 constitutional amendment that put an end to 'double track' administration has been wrongly overshadowed by the 1929 amendment that modified the role of the federal president.

Keywords: administration - Austrian Federal Constitution 1920 -

constitutional amendment 1925 - governor - Office of the Provincial Government

\section{Allgemeines}

Die „Doppelgleisigkeit der Verwaltung“ ist ein rechtshistorischer Tiefwurzler. Das Nebeneinander staatlicher „allgemein-politischer" Verwaltung und autonomer Landesverwaltung führt weit zurück in den frühneuzeitlichen „institutionellen Dualismus" von landesfürstlichen und landständischen Behörden. ${ }^{1}$ Es erfasst verschiedene territoriale Ebenen, seit eine monarchische Länderverbindung $\mathrm{zu}$ einem dezentralisierten Gesamtstaat gestaltet wurde. Letztlich steht es für das erfolgreiche Konzept „jahrhundertelange[r] dynastische[r] Arbeit“2, außerhalb der Verfassung des Heiligen Römischen Reiches einen Schirm über die reichsverfassungsrechtliche Ländervielfalt zu spannen und durch Etablierung eines modernen Verwaltungsapparats ei-

\footnotetext{
${ }^{1}$ BRAUNEDER, Verfassungsgeschichte 64.

${ }^{2} \mathrm{KOCH}$, Frage 60; vgl. auch KeLSEN, Vollendung 303.
}

nen Staat zu begründen, in dem Reste landständischen Einflusses zwar als autonome Landesverwaltung überlebten, aber nur noch ein Schattendasein fristeten. ${ }^{3}$ Zum Problem wurde diese „Doppelgleisigkeit“ erst im Konstitutionalismus des 19. Jahrhunderts durch das Nebeneinander regulierter Landesautonomie (Landesordnungen 1861) und neu organisierter „politischer Verwaltungsbehörden" $1868^{4}$ - nicht zuletzt vor dem Hintergrund einer auf allen Ebenen zunehmenden Gesetzgebungs- und Verwaltungsaktivität. Trotz vielfältiger Gestaltungsideen blieb das Problem bis zum Untergang Österreich-Cisleithaniens ungelöst. ${ }^{5}$ Der „Dualismus von (dezentraler) autonomer und (zentraler) landesfürstlicher Verwaltung charakterisiert die Ver-

\footnotetext{
${ }^{3}$ BRAUnEDER, Verfassungsgeschichte 101-103; DERS., Geschichte.

${ }^{4}$ RGBl. 1868/44.

${ }^{5}$ WiEDERIN, Diskussion; vgl. Vollversammlung; MERKL, Länderautonomie 69-71.
} 
waltungsorganisation im Bereich des Landes zur Zeit der Monarchie“", so schrieb Hans Kelsen. ${ }^{6}$

In ähnlicher Weise gehört die mittelbare Bundesverwaltung7 „zu jenen Einrichtungen, die dem österreichischen Verwaltungsaufbau ihre typische Gestalt geben“, zu den „staatsrechtlichen Eigentümlichkeiten der österreichischen Bundesverfassung“, zu den „wesentlichsten Elemente[n] der österreichischen Bundesstaatlichkeit" ${ }^{9}$ Ein „klarer, einfacher und gleichzeitig schlanker Verwaltungsaufbau“ würde konzentrierte und ökonomische Verfahrensabwicklung ermöglichen. Die Behörden wären bürgernah, leicht erreichbar und mit den regionalen Verhältnissen vertraut; damit würde auch die Akzeptanz ihrer Entscheidungen gefördert. ${ }^{10} \mathrm{Um}$ gekehrt könne die „Bundesebene“ auf einen „zeit- und kostenintensiven Auf- und Ausbau eigener Verwaltungsstrukturen verzichten".11 Insgesamt wird die mittelbare Bundesverwaltung als ein "Strukturmerkmal "12 nicht nur des österreichischen Bundesstaates charakterisiert. Als ein „systemrelevantes Strukturelement der österreichischen Verfassung“ begrenzt sie die Übertragung erstinstanzlicher Aufgaben an die Bundesministerien; der Landeshauptmann darf als Träger der mittelbaren Bundesverwaltung nicht ausgeschaltet werden. ${ }^{13}$

Beide Eckpunkte des Themas wurden und werden also für das jeweilige Staatswesen als charakteristisch empfunden. Ihre Verbindung erfor-

\footnotetext{
${ }^{6}$ KELSEN, Vollendung 305.

${ }^{7}$ WEBER, Bundesverwaltung.

8 JABLONER, Bundesminister, folgt FrOEHLICH, Eigentümlichkeiten 81, 85 (,ganz uns eigentümliche [...] Einrichtung“).

${ }^{9}$ WEBER, Bundesverwaltung 7.

${ }^{10}$ RANACHER, Verwaltung 200-202.

${ }^{11}$ BUßJÄGER, Kontrolle $5 \mathrm{f}$.

12 Ebd.

${ }^{13}$ GAMPER, Systemrelevanz 557; exemplarisch HOLZER, Weinaufsicht 126ff; vgl. weiters HöLLBACHER, Kehrseite 88ff; HOlZINGER, Fragen 77f; PURTSCHER, Organisationshoheit 338f; STEINER, Landeshauptmann $402 \mathrm{ff}$.
}

dert zunächst einen Blick auf die einzelnen Phasen der Entwicklung (II.), bevor sodann einige intensiv diskutierte Problembereiche skizziert werden (III.) Dabei sollen insbesondere zeitgenössische Literaturstimmen zu Wort kommen. ${ }^{14}$

\section{Phasen}

\section{Phase: Vor 1918}

Vor 1918 gab es in den österreichischen Ländern zwei verschiedene Verwaltungsapparate, die als „Landes"-Verwaltung bezeichnet wurden. ${ }^{15}$ Die (gesamt)staatliche (historisierend auch „landesfürstliche“) Verwaltung war zentralistisch organisiert. Dabei gab es unterhalb der Zentralbehörden (Ministerien seit 1848) staatliche Mittelbehörden, meist Statthaltereien genannt, in kleineren Verwaltungssprengeln aber auch „Landesregierung“; der höchste Beamte („Landeschef“) war hier der Statthalter bzw. der „Landespräsident". ${ }^{16}$ Die Komposita mit „Land“ hatten ihren Grund darin, dass als Sprengel für diese mittlere staatliche Verwaltungsebene in aller Regel die historischen Länder fungierten. Nur vereinzelt lebte jene länderübergreifende („Provinzial“-) Struktur weiter, die seit der Mitte des 18. Jahrhunderts als "Gouvernementsbezirke“ gerade gegen die landständisch geprägten Länder etabliert worden war: Auf dem heutigen Staatsgebiet Österreichs war dies bei den Ländern Tirol und Vorarlberg der Fall, die gemeinsam einen Statthaltereisprengel bildeten. ${ }^{17}$ Nicht breit durchgesetzt hat sich die Terminologie von Ulbrich, der die gesamtstaatlichen Behörden im Land als „Territorialbehörden“ oder „obrigkeitliche Behörden innerhalb der einzelnen Länder" bezeichnete. ${ }^{18}$

\footnotetext{
14 Vgl. dazu KoHL, Prinzip 117ff.

${ }^{15}$ Vgl. etwa HeLlbling, Landesverwaltung.

${ }^{16}$ RGBl 1868/44.

${ }^{17} \mathrm{Vgl}$. UlbRICH, Staatsrecht $143 \mathrm{f}$.

${ }^{18}$ Ebd. 143.
} 
Unabhängig von der staatlichen Verwaltung gab es eine - quantitativ weniger bedeutende - , autonome" Verwaltung der Länder. Geleitet wurde sie vom „Landesausschuss", dem „verwaltende[n] und ausführende[n] Organ der Landesvertretung ", 19 also einem Exekutivausschuss des Landtages unter der Leitung des „Landeshauptmannes" (teils auch "Landmarschalls"). Abgesehen von wenigen Ausnahmen - etwa dem Gemeinde-, Bau- oder Armenwesen - beschäftigte sich die autonome Landesverwaltung mit Angelegenheiten, die eher der Wirtschaftsals der Hoheitsverwaltung zuzurechnen waren. ${ }^{20}$ „Das Land ist zunächst ein Kommunalverband höchster Ordnung, der unter Verwendung eigenen Vermögens durch seine eigenen Behörden (Landtag und Landesausschuss) die Selbstverwaltung sozialer Wohlfahrtsaufgaben besorgt", so fasste dies Ulbrich zusammen. ${ }^{21}$ Mit „obrigkeitlicher, als Ausübung der Staatsgewalt erscheinender Macht" erschienen die autonomen Landesbehörden dann, wenn sie zur Finanzierung ihrer Aufgaben Zuschläge zu den staatlichen Steuern anordneten (,Auflagengewalt") oder eine "Aufsichtsgewalt über die unteren Kommunalverbände" ausübten. ${ }^{22}$

Den Kern der autonomen Landesverwaltung bildeten also Reste ständischer Selbstverwaltung, die im Gefolge der Landesordnungen $1861^{23}$ in den Sog zunehmender Regulierungsaufgaben gerieten. Im Gegensatz zur kontinuierlich ausgebauten landesfürstlich-monarchischen Verwaltung bestand eine nennenswerte Kontinuität zum Spätmittelalter oder zur Frühneuzeit nach den absolutistischen Reformen aber nicht mehr. Dessen ungeachtet wurde auch zur autonomen Landesverwaltung gelegentlich rechtshistorisch argumentiert, wenngleich unzutreffend. So be-

\footnotetext{
${ }^{19} \S 11$ Landesordnung NÖ, RGBl. 1861/20, Beilage 2a.

${ }^{20}$ ADAMOVICH, Reform 245; PeNDL, Frage 117.

${ }^{21}$ UlBRICH, Staatsrecht 161.

22 Ebd.

${ }^{23}$ ROSNer, MOTZ-LinHART, 1861.
}

hauptete Pendl, dass das „Anwachsen der landesherrlichen Gewalt [...] eine kräftigere Entwicklung der Landstände [...] zur Folge“" gehabt hätte ${ }^{24}$ - obwohl doch eher das Gegenteil zutraf. Mit der Behauptung, das „Nebeneinander von staatlicher Verwaltung und von autonomer Landesverwaltung“ hätte sich „,in historischer Notwendigkeit entwickelt", erwies sich Pendl übrigens als Anhänger einer naturalistischen Rechtswissenschaft im Sinne darwinistischer Ideen. ${ }^{25}$

\section{Phase: $1918-1920$}

Die zweite Phase setzte nicht sogleich mit der Staatsgründung Deutschösterreichs am 30. Oktober $1918^{26}$ ein, sondern erst mit dem „Gesetz betreffend die Übernahme der Staatsgewalt in den Ländern" vom 14. November 1918.27 Dieses Gesetz wurde in der Folge vielfach als Wendepunkt genannt, an dem der Weg vom Einheitsstaat zum Bundesstaat begann. ${ }^{28}$ Dabei erschien es bemerkenswert, dass das „folgenreiche, wenig prinzipienklare [...] Gesetz [...], aus zentralistischen Träumen erwachend, die Staatsgewalt in den Ländern in die Gewalt der Länder" brachte, ${ }^{29}$ dass also der Effekt des Gesetzes nicht jenen Absichten des Gesetzgebers entsprach, die der Name andeutet: „Die praktische Wirkung des Gesetzes, das eine Übernahme der Staatsgewalt in den Ländern intendierte, war die Übergabe

\footnotetext{
${ }^{24}$ PendL, Frage 116.

${ }^{25}$ Ebd. 117; vgl. zum Recht, das werden musste, Arbeitsgemeinschaft Österreichische Rechtsgeschichte, Manual 250.

${ }^{26}$ Dazu BRAUNEDER, Deutschösterreich 16ff.

${ }^{27}$ StGBl. 1918/24; zu den Ländern im Staatsgründungsprozess Arbeitsgemeinschaft Österreichische Rechtsgeschichte, Manual Rz. 1814-1818; ausführlich BRAUNEDER, Deutschösterreich 272ff.

${ }^{28}$ KELSEN, Vollendung 302: Bundesstaat als sachlich unbegründetes Produkt des "Zufall[s]“ eines „Geburtsfehler[s] bei der erstmaligen Konstruktion Deutschösterreichs“; vgl. auch DERS., Stellung 104ff.; MERKL, Staat 1f.; OFNER, Bundesverfassung 67. ${ }^{29}$ WITTMAYER, Verfassungsentwicklung 458.
} 
dieser Staatsgewalt an die Länder", so formulierte es Kelsen in Anlehnung an Merk1. ${ }^{30}$

Von den zahlreichen Fragen, die dieses Gesetz aufwirft, kann hier nur die Umgestaltung der Verwaltungsstruktur weiterverfolgt werden. Auf der Ebene der Länder wurden die Landtage durch politisch breiter legitimierte provisorische Landesversammlungen ersetzt, die das Kräfteverhältnis der politischen Parteien nach der Reichsratswahl 1911 widerspiegelten; an die Stelle der Landesausschüsse traten (von den provisorischen Landesversammlungen gewählte) „Landesräte“ für die autonome Landesverwaltung. Daneben hatten die provisorischen Landesversammlungen aber auch einen „Landeshauptmann" sowie dessen Stellvertreter zu wählen, die in ihrer Gesamtheit die „Landesregierung" bildeten. Dabei erfolgte die Wahl des Landeshauptmanns mit Stimmenmehrheit, jene der Stellvertreter nach dem Verhältniswahlrecht. ${ }^{31}$ An diese Landesregierung gingen die Agenden der früheren Landeschefs sowie der dazu vorhandene Verwaltungsapparat über; nur die Landeschefs selbst wurden ihres Amtes enthoben. So wie früher der Statthalter dem k.k. Minister, so war nun die Landesregierung dem deutschösterreichischen Staatssekretär untergeordnet. Daher war die Landesregierung auch „bei ihrer gesamten Amtsführung an die Dienstanweisungen der deutschösterreichischen Staatsregierung gebunden". 32

Plakativ und in „lakonischer Kürze“33 formulierte das Gesetz: „Die Teilung der öffentlichen Verwaltung in landesfürstliche und autonome ist aufgehoben." ${ }^{34}$ Tatsächlich blieben aber getrenn-

\footnotetext{
${ }^{30}$ KELSEN, Stellung 104; MERKL, Erläuterungen 103.

${ }^{31}$ KLEIN, Verhältniswahl 97ff.; PESENDORFER, Landeshauptmann $19 \mathrm{f}$.

32 \& 8 StGBl. 1918/24.

33 ADAMOVICH, Reform 246.

${ }^{34} \S 9$ StGBl. 1918/24. Darin zeigt sich der Wunsch, „das Doppelgeleise der Verwaltung, dessen Übelstände im alten Österreich genugsam beklagt wurden“ (KELSEN, Stellung 114) zu überwinden.
}

te Apparate bestehen: "Gleich als ob aber das Gesetz selbst den von ihm feierlich deklarierten Grundsatz der Vereinheitlichung des Verwaltungsapparates in den Ländern nicht allzu ernst nehmen wollte, sah es sofort wieder zunächst eine provisorische Organisation vor, in der der Gedanke der Einheitlichkeit der Landesverwaltung überhaupt nicht mehr wieder zu erkennen war: bis zur Durchführung der entsprechenden Verwaltungsreform sollten nämlich in jedem Lande die Geschäfte der ehemals politischen Landesbehörde von jenen der autonomen Landesbehörde getrennt geführt werden, und zwar Erstere von staatlichen Beamten unter Leitung der anstelle der Statthalter (Landespräsidenten) berufenen kollegialen Landesregierungen, Letztere von autonomen Landesbeamten unter Leitung der an die Stelle der ehemaligen Landesausschüsse tretenden Landesräte." ${ }^{35}$ Nur an der Spitze existierten Verknüpfungen: Der Landesrat wählte „,zur Vereinfachung und Vereinheitlichung des Dienstbetriebes" einen Landesamtsdirektor, ${ }^{36}$ der, dem Landeshauptmann persönlich zugeteilt, als Vorgesetzter aller Beamten fungierte - sowohl jener des Landesrates als auch jener der Landesregierung. Personell verflochten waren auch Landesregierung und Landesrat, weil hier wie da der Landeshauptmann den Vorsitz führte. ${ }^{37}$

Eine Ausnahme von dieser Verwaltungsstruktur bildete Vorarlberg, das vor 1918 kein eigener Verwaltungssprengel mittlerer Ebene gewesen war; der Sprengel der in Innsbruck gelegenen Statthalterei hatte sich auf Tirol und Vorarlberg erstreckt. Als nun durch das Gesetz vom 14. November 1918 betreffend die Übernahme der Staatsgewalt in den Ländern auch in Vorarlberg eine Landesregierung mit der staatlichen Verwaltung betraut wurde, war kein Statthalter zu

\footnotetext{
35 ADAMOVICH, Reform 247.

${ }^{36}$ HERRNRITT, Verwaltungsrecht 75; SCHMITZ, Landesamtsdirektor.

${ }^{37}$ KELSEN, Stellung 103.
} 
ersetzen; die Landesregierung konnte nicht einfach auf einen bestehenden Behördenapparat zurückgreifen. In dieser Situation verzichtete man darauf, eine eigene staatliche Behörde neu einzurichten, sondern schuf sogleich ein einheitliches Amt, in dem unter der Leitung des Landeshauptmannes Staatsbeamte und Landesbeamte nebeneinander tätig wurden. ${ }^{38}$

\section{Phase: $1920-1925$}

Das B-VG 1920 änderte die Verwaltungsstruktur in zwei wesentlichen Aspekten. Einerseits wurde die gesamtstaatliche „Bundesverwaltung“ vom Kollegialorgan „Landesregierung“ auf das monokratische Organ "Landeshauptmann" übertragen. ${ }^{39}$ Andererseits war damit die Existenzberechtigung einer vom Landesrat gesonderten Landesregierung entfallen. Der Begriff „Landesregierung“ konnte nun für die Führung der autonomen Landesverwaltung verwendet werden; Landesrat und Landesregierung erschienen somit „verschmolzen“. Die Landesregierung hatte den ihr seit 1918 unterstehenden gesamtstaatlichen Verwaltungs- und Beamtenapparat jedoch nicht in diese "Verschmelzung“ mitgerissen. Es blieb beim Nebeneinander von zwei Verwaltungsbereichen, nun als „mittelbare Bundesverwaltung“ bzw. „selbstständige Landesverwaltung".

Eine Verknüpfung ergab sich wieder durch den Landeshauptmann und die in ihm verkörperte „Personalunion zweier ganz verschiedener Wirkungsbereiche“: Einerseits „,ist er das Oberhaupt des Gliedstaates“. ${ }^{40}$ Als solches ist er der Vorsitzende der Landesregierung und damit das oberste Organ der Vollziehung im selbstständigen Wirkungsbereich des Landes und nur diesem verantwortlich. Andererseits aber ist er persönlich - unter Mitwirkung der ihm unterstell-

\footnotetext{
38 ADAMOVICH, Frage 54f.

39 PURTSCHER, Organisationshoheit 339.

${ }^{40} \mathrm{Vgl}$. FroEHLICH, Landeshauptmann 135 („überragende[...] Stellung [...] als Gliedstaatshaupt" ${ }^{\prime \prime}$.
}

ten Landesbehörden - durch die Verfassung auch mit der mittelbaren Bundesverwaltung betraut. In dieser Funktion ist er ein Bundesorgan und wie alle anderen Bundesorgane in die Organisation der Bundesverwaltung, also in die Hierarchie der mit der Bundesvollziehung betrauten Stellen eingeordnet". ${ }^{41}$ Der Landeshauptmann war also der Bundesregierung unterstellt und vor dem Verfassungsgerichtshof verantwortlich. ${ }^{42}$

Besondere Bedeutung hatte es, dass die mittelbare Bundesverwaltung durch den „Landeshauptmann und die ihm unterstellten Landesbehörden ${ }^{\prime 43}$ auszuüben war. Daraus konnte man ableiten, dass sich der Landeshauptmann zur Vollziehung in Angelegenheiten der mittelbaren Bundesverwaltung jeder Landesbehörde bedienen konnte, dass also keine speziellen Behörden für die mittelbare Bundesverwaltung einzurichten oder aufrechtzuerhalten waren. ${ }^{44}$ Auch die Funktion des Landesamtsdirektors gab es weiterhin.45 Das im B-VG genannte „Amt der Landesregierung“ erschien jedoch als „ein in seiner Bedeutung höchst problematisches Programm"46 oder gar als „ein äußerst gefährliches Experiment auf dem Gebiete der Verwaltungsreform" ${ }^{4}{ }^{47}$

Als „Programm“ konnte die mittelbare Bundesverwaltung deshalb bezeichnet werden, weil die Verwaltungsstrukturfragen infolge des Verfassungs-Übergangsgesetzes 1920 bis zum Inkrafttreten eines besonderen Verfassungsgesetzes über die Organisation der Verwaltung in den Ländern in der Schwebe blieben. ${ }^{48}$ Damit wurde ein Teil des vom B-VG vorgefundenen Zustandes prolongiert: „Durch das Übergangsgesetz ist

${ }^{41}$ Ebd. 132.

${ }^{42}$ KELSEN, Vollendung 305f.

${ }^{43}$ Art. 102 Abs. 1 B-VG.

${ }^{44}$ ADAMOVICH, Frage 47.

${ }^{45} \mathrm{Vgl}$. Art. 106 B-VG.

${ }^{46}$ AdAmovich, Frage 48, 51f.; PENDL, Frage 106.

${ }^{47}$ Ebd. 118.

${ }^{48} \S \S 8,42$ Abs. 2 V-ÜG; Adamovich, Frage 48, 51f.; PENDL, Frage 106. 
also das Doppelgeleise in der Verwaltung der Länder, das durch das Bundes-Verfassungsgesetz in radikaler Weise beseitigt werden sollte, geradezu in Umkehrung dieses Grundsatzes ausdrücklich belassen worden“. ${ }^{49}$ Für den Übergangszeitraum bis zum endgültigen Wirksamwerden der Verfassung 1920 existierten in den Ländern jeweils zwei getrennte Behördenapparate. ${ }^{50}$ In den Zuständigkeitsbereich der Landesverwaltung fielen dabei jene Gegenstände, die beim Inkrafttreten des B-VG (10. November 1920) von den ehemals autonomen Landesbehörden verwaltet wurden; alle übrigen Angelegenheiten waren provisorisch Bundessache..$^{51}$

Für das Inkrafttreten der Kompetenzbestimmungen $^{52}$ des B-VG 1920 waren drei Bedingungen - jeweils in Form eines Verfassungsgesetzes - zu erfüllen: Einerseits bedurfte es einer Regelung der finanziellen Beziehungen zwischen Bund und Ländern (wie sie 1922 durch das Finanzverfassungsgesetz erfolgte), andererseits einer Ordnung der Wirkungsbereiche ,auf dem Gebiet des Schul-, Erziehungs- und Volksbildungswesens".53 Schließlich sollte ein Verfassungsgesetz die „Organisation der allgemeinen staatlichen Verwaltung in den Ländern" regeln. ${ }^{54}$ Dieses Verfassungsgesetz war somit eine Vorwegnahme der im B-VG als Gegenstand der Bundesgrundsatzgesetzgebung definierten „Organisation der Verwaltung in den Ländern". ${ }^{55}$ Als Verfassungsgesetz hätte es, wie Adamovich nahezu hoffnungsvoll in den Raum stellte, "die Organisation der Landesverwaltung auch in völliger Abkehr von diesen Grundsätzen [des Art.

\footnotetext{
${ }^{49}$ So ADAMOVICH, Frage 50.

50 § 42 V-ÜG; ADAMOVICH, Reform 248.

${ }^{51}$ ADAMOVICH, Reform 229.

52 Vgl. dazu MerKL, Problem.

${ }^{53} \S 42$ Abs. 1 V-ÜG; vgl. ADAMOvICH, Reform 229.

${ }^{54}$ Ebd.

${ }^{55}$ Art. 12 Abs. 1 B-VG (stand infolge der aufgeschobenen Wirksamkeit der Kompetenzbestimmungen noch nicht in Kraft).
}

102 B-VG] gestalten“ können. ${ }^{56}$ Das schließlich erlassene „Bundesverfassungsgesetz betreffend Grundsätze für die Einrichtung und Geschäftsführung der Ämter der Landesregierungen außer Wien ${ }^{\prime 57}$ sollte diese Hoffnung aber nicht erfüllen.

Zeitgenössisch war zunächst nur eine kurze Übergangsfrist von wenigen Monaten erwartet worden, $^{58}$ aufgrund der Parteiengegensätze wurden daraus fast fünf Jahre. Mehrere Ursachen waren dafür ausschlaggebend, dass es aber schließlich doch zur Neuorganisation der Verwaltung kam.

Zunächst ist das Kostenargument zu nennen. Dies zeichnete sich schon im Wiederaufbaugesetz vom 27. November $1922^{59}$ ab: Gegenüber den Geldgebern der Genfer Anleihe wurde als Teil des Sparprogramms eine „Verschmelzung des Apparates der ehemals autonomen Verwaltung in den Ländern mit jenem der staatlichen Verwaltung" angekündigt.60 In diesem Sinne charakterisierte Merkl die Reform durch den „erklärten Zweck, durch Einsparung von Verwaltungsaufwand der Lösung des Finanzproblems zu dienen“; er sah darin eine "Metastase des Finanzproblems auf das Gebiet der Verwaltung“. ${ }^{11} \mathrm{Ob}$ dieser Zweck eines „Ersparungserfolges" erreicht werden könne, blieb strittig; aus praktischer Sicht warnte Pendl vor einem „weit überschätzten, tatsächlich nur im geringen Maße erzielbaren" Effekt. ${ }^{62}$

Ein weiterer Grund lag in den negativen Folgen des Weitergeltens der Kompetenzbestimmungen von 1867. Viele seit damals neu entstandene Materien fielen nach der Generalklausel des $§ 12$

\footnotetext{
${ }^{56}$ ADAMOVICH, Frage 49.

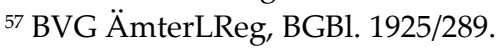

58 ADAMOVICH, Reform 230.

${ }^{59}$ BGBl. 1922/843; vgl. KOHL, Gesetzgebung 318ff (321).

${ }^{60}$ AdAmovich, Reform 249; Kelsen, Vollendung 309.

${ }^{61}$ MERKL, Verwaltungsreform 164, macht damit den engen Zusammenhang zwischen 1925 und 1922 deutlich. 62 PENDL, Frage 118.
} 
StGG-Reichsvertretung den Ländern zu, auch wenn dies sachlich unsinnig war. Besonders diskutiert wurde dies im Zusammenhang mit einem Erkenntnis des Verfassungsgerichtshofes, wonach für die gesetzliche Krankenversicherung der Landarbeiter die Landesgesetzgebung zuständig war. ${ }^{63}$

Schließlich wurde die Reform von 1925 dadurch angetrieben, dass sich die Organisation der Landesverwaltung seit 1920 in verschiedenen Ländern unterschiedlich entwickelte. ${ }^{64}$ Anknüpfungspunkt war dabei die bereits erwähnte Verlagerung der staatlichen Verwaltung von den Landesregierungen $\mathrm{zu}$ den Landeshauptmännern. Schon „bald nach dem Wirksamkeitsbeginn [des B-VG] zeigten sich nun in einzelnen Ländern Bestrebungen, die auf eine Teilnahme der übrigen Mitglieder der Landesregierung an der Führung der mittelbaren Bundesverwaltung im Lande abzielten“. Einzelnen Landesregierungsmitgliedern wurde jeweils im Auftrag, unter Aufsicht und Verantwortlichkeit des Landeshauptmannes eine Übernahme von Aufgaben der mittelbaren Bundesverwaltung gestattet. ${ }^{65}$ Damit überschattete die Regelung von 1918 jene des B-VG 1920, zugleich zeichnete sich bereits die Novelle $1925 \mathrm{ab}$.

Die regionale Differenzierung der Verwaltungsstruktur hatte ja eigentlich schon vor 1920 durch den Sonderfall Vorarlbergs begonnen. Sie setzte sich in Wien fort, wo der Magistrat aufgrund des V-ÜG neben den Agenden der Gemeindeverwaltung und der politischen Bezirksverwaltung auch "die Funktionen einer politischen Landesbehörde übernahm", ${ }^{66}$ sodass er im Ergebnis bereits als einheitliches Amt der Landesregierung bezeichnet werden könnte. Den nächsten Ausnahmefall verursachte der Erwerb des Burgenlandes. Hier übte der Bund die ge-

\footnotetext{
${ }^{63}$ ADAmOvich, Reform 231 (VfGH 27. 6. 1934, G 2/24).

${ }^{64}$ Ebd. 249.

${ }^{65}$ ADAMOVICH, Reform 242.

${ }^{66} \S 33$ V-ÜG; vgl. ADAMOVICH, Reform 248.
}

samte Vollziehung aus.67 1922 wurde ein Amt der Landesregierung geschaffen, das sowohl den selbstständigen Wirkungskreis des Landes als auch die mittelbare Bundesverwaltung besorgte. ${ }^{68}$ Daraus resultierte eine im Vergleich zum B-VG umgekehrte Konstellation: Der Landeshauptmann war im selbstständigen Wirkungsbereich des Landes verpflichtet, die Beschlüsse der Landesregierung auszuführen. ${ }^{69}$ In anderen Ländern (Vorarlberg, Salzburg, Kärnten) kam es zu einer materiellen Vereinheitlichung der Verwaltung, indem man in beiden Wirkungsbereichen parallele Strukturen schuf, auf deren Basis die Agenden leichter konzentriert werden konnten. ${ }^{70}$

Als problematisch erwies es sich auch, dass das B-VG zwar „eine vom Landtag zu wählende Landesregierung“ vorsah, deren Ausgestaltung jedoch den Landesverfassungen überließ. Rasch zeigten sich daher erhebliche Unterschiede etwa in der Zusammensetzung der Landesregierungen $^{71}$ - eine bedenkliche Entwicklung, weil die Mitglieder der Landesregierungen vom Bund besoldet wurden. Die Länder entschieden also über die vom Bund zu tragenden Kosten. So wurde die Vergrößerung der Landesregierung in der Steiermark kritisiert: Hier war, obwohl mit der Untersteiermark rund ein Drittel des Landes verloren gegangen war, die Zahl der Landesregierungsmitglieder auf 11 (im Vergleich zu ehemals 7 Landesausschussmitgliedern) sogar um die Hälfte erhöht worden. ${ }^{72}$

Auch der Wahlmodus war den Ländern überlassen. Überwiegend wurde die Landesregierung

\footnotetext{
${ }^{67} \S 4$ Abs. 1 BVG über das Burgenland vom 25. 1. 1921, BGBl. 1921/85.

${ }^{68}$ BVG vom 7. 4. 1922, BGBl. 1922/202; ADAMOVICH, Reform 248.

${ }^{69}$ Dazu Ders., Frage 54f.

${ }^{70}$ DERS., Reform 248f; DERS., Frage 53-56; zur Parallelführung von Geschäftsgruppen ablehnend PENDL, Frage 107.

${ }^{71}$ Vgl. dazu AdAMOVICH, Frage.

72 PENDL, Frage 105.
} 
nach dem Verhältniswahlrecht gewählt; man verstand sie demnach als Äquivalent des historischen Landesausschusses, als Vollzugsorgan des Landtages. Nur Wien und Vorarlberg sahen ein Mehrheitswahlrecht vor, woraus in Wien die Unterscheidung in "amtsführende“ und „nicht amtsführende" Stadträte resultiert. ${ }^{73}$ Das Proportionalwahlrecht erschien Adamovich übrigens nur als Übergangsphänomen: Wenn „nach dem Inkrafttreten der Kompetenzbestimmungen [des B-VG] auch weite Gebiete der Hoheitsverwaltung in den selbstständigen Wirkungsbereich der Länder übergehen werden, dann wird sich die dringende Notwendigkeit einheitlich gefügter Regierungen auch in den Ländern mit aller Bestimmtheit erweisen und werden die Bestimmungen der Landesverfassung über die Wahl der Mitglieder der Landesregierungen sicherlich einer Revision unterzogen werden müssen". ${ }^{74}$ Diese Prognose erfüllte sich nicht; erst in den letzten Jahren wurde der Proporz stärker hinterfragt.

Diese Regelungsvielfalt machte aus Sicht des Bundes eine Vereinheitlichung der Strukturen notwendig. ${ }^{75}$ Damit eng verknüpft war eine Neuordnung des Verfahrensrechts; beide Fragen standen unter dem Einfluss der notwendig gewordenen Einsparungen im Verwaltungsapparat. Prägnant formulierte Merkl: „Der Beamtenabbau bedingt also eine Parallelaktion: den Arbeitsabbau. Dieser kann sich - und muss sich, wenn er wirkungsvoll sein soll - auf doppelte Weise äußern: als Verminderung der Verwaltungsagenden nicht weniger denn als Vereinfachung der Verwaltungsmethoden." ${ }^{16}$ Vor 1925 hatte der Verwaltungsgerichtshof aus unzähligen Einzelnormen ein Verwaltungsverfahrensrecht entwickelt, das zur Grundlage der Kodifi-

\footnotetext{
${ }^{73}$ Dazu Adamovich, Frage 36-39.

${ }^{74}$ Ebd. 39.

${ }^{75}$ ADAMOVICH, Reform 249.

${ }^{76}$ MERKL, Verwaltungsreform 170.
}

kation wurde. ${ }^{77}$ Obwohl ein neues Verfahrensrecht also eine zweckmäßige Begleitmaßnahme zur neuen Verwaltungsorganisation war, stand letztere ersterem auch im Weg: Art. 11 B-VG, wonach Verwaltungsverfahrensgesetzgebung dem Bund zufiel, war bei Fertigstellung der Verwaltungsverfahrensgesetze noch gar nicht in Kraft. Daher bedurfte es einer ,spezielle[n] verfassungsrechtliche[n] Grundlage für die Verwaltungsverfahrensreform [...] durch ein eigenes Bundesverfassungsgesetz vom 21. Juli 1925“.78 „,Die mit der Verfassungsreform verknüpfte und ebenfalls als Bollwerk gegen die Zersplitterung der Verländerung gedachte Verwaltungsreform (Verfahrensreform) bedeutet erstmalige, bis in jede Einzelheit vom ökonomischen Prinzip beherrschte gesetzliche Gesamtregelung des Verfahrens bei einem Großteil der Verwaltungsbehörden." 79

Neben sachlichen Gründen war für die Verfassungsnovelle 1925 auch eine politische Interessenverschiebung bedeutsam. Die Christlichsoziale Partei galt nicht mehr als „Repräsentantin der föderalistischen Strömungen", weil sie im „Parteiinteresse“ Wien nicht „zu einem gar zu eigenmächtigen Staat im Staate werde[n]" lassen durfte. Umgekehrt mussten die Sozialdemokraten ihre traditionell zentralistische Konzeption im Interesse möglichst weitgehender Selbstständigkeit Wiens überwinden. ${ }^{80}$

\section{Phase: Ab 1925}

1925 traten die Kompetenzbestimmungen des BVG in Kraft. ${ }^{81}$ Dies reduzierte die Vollziehung des Bundes auf die ausdrücklich genannten Bereiche der „unmittelbaren Bundesverwaltung“.

\footnotetext{
77 MANNLICHER, Verwaltungsreform 364f.

78 BGBl. 1925/271; MANNLICHER, Verwaltungsreform 361.

79 WITTMAYER, Verfassungs- und Verwaltungsreform 486.

${ }^{80}$ MerKL, Epilog 1242; WitTMAYER, Verfassungsentwicklung $458 \mathrm{f}$.

${ }^{81}$ Vgl. ADAMOVICH, Verfassungs- und Verwaltungsreform; DERS., Typen.
} 
Die Kompetenzverschiebung charakterisierte Merkl als „ganz außerordentliche Erweiterung der Länderkompetenz auf dem Gebiete der Verwaltung"82, wobei ihm die mittelbare Bundesverwaltung als „dunkelste[r ...] Punkt der Verfassungsreform" erschien. ${ }^{83}$

Dabei machte die B-VG-Novelle im engeren Sinn ${ }^{84}$ ein schon seit 1920 vereinzelt praktiziertes Modell zur Regel: Die Landesregierung konnte nun „bei Aufstellung ihrer Geschäftsordnung beschließen, daß einzelne Gruppen von Angelegenheiten der mittelbaren Bundesverwaltung wegen ihres sachlichen Zusammenhanges mit Angelegenheiten des selbständigen Wirkungskreises des Landes [...] von Mitgliedern der Landesregierung zu führen sind“. ${ }^{85}$ Deren Tätigkeit für die mittelbare Bundesverwaltung erfolgte zwar „im Namen des Landeshauptmannes“, dieser hatte jedoch juristisch geringen Einfluss darauf, weil ihm - wie jedem anderen Mitglied der Landesregierung - nur eine Stimme zukam. Theoretisch konnte die Landesregierung solche Agenden also mit Stimmenmehrheit gegen den Landeshauptmann verteilen. Auch der Bund konnte nicht beeinflussen, wie das Land die Struktur der mittelbaren Bundesverwaltung ordnete. Ansprechpartner der Bundesregierung und Adressat ihrer Weisungen war daher weiterhin der Landeshauptmann, der diese Weisungen an das zuständige Landesregierungsmitglied weiterzugeben hatte. Er war verpflichtet, zur Durchführung der auf dem Gebiet der mittelbaren Bundesverwaltung erhaltenen Weisungen auch jene Mittel einzusetzen, über die er als Organ des selbstständigen Wirkungsbereichs des Landes verfügte.86 Den Landeshauptmann

\footnotetext{
${ }^{82}$ MERKL, Grundlagen 221.

${ }^{83}$ DERS., Epilog 1269.

${ }^{84}$ BVG vom 30. 7. 1925, BGBl. 1925/268; vgl. ADAMOVICH, FroEHLICH, Novellen.

${ }^{85}$ Art. 103 B-VG.

${ }^{86}$ Art. 103 Abs. 1; zustimmend ADAMOVICH, Reform 240. Dies war Konsequenz der „Verschmelzung der
}

traf dafür die „Ministerverantwortlichkeit“ nach Art. 142 B-VG. ${ }^{87}$ In ,"seiner Eigenschaft als Organ der mittelbaren Bundesverwaltung" wurde der Landeshauptmann nunmehr durch einen von der Landesregierung bestimmten Landeshauptmannstellvertreter vertreten. ${ }^{8}$

Das neue Verwaltungsverfahrensrecht erschien als ausreichende Vorsichtsmaßnahme gegen zu große Macht des Landeshauptmannes: Als „nachgeordnetes Organ fällt er jetzt auch unter die neue Vorschrift, nach der nachgeordnete Organe die Befolgung einer Weisung nur dann ablehnen können, wenn sie von einem unzuständigen Organ erteilt wurde oder die Befolgung gegen strafgesetzliche Vorschriften verstoßen würde“ .89

Die Neuorganisation der Verwaltung, die „zweite Gruppe des Reformwerks",90 erfolgte teils im Rahmen der Übergangsgesetzgebung, ${ }^{91}$ teils in einem besonderen Bundesverfassungsgesetz vom 30. Juli 1925 ,,betreffend Grundsätze für die Einrichtung und Geschäftsführung der Ämter der Landesregierungen außer Wien".92 Dieses Gesetz war mit der B-VG-Novelle im engeren Sinn dadurch verknüpft, dass die „Einrichtung der Ämter der Landesregierungen mit dem Zeitpunkte des Inkrafttretens [der Kompetenzartikel des B-VG] durchzuführen" war. ${ }^{93}$

Diese Ämter der Landesregierung, zuvor „,noch reine Bundesbehörde[n]",94 sowie die ihnen un-

Amtsapparate“ zum Amt der Landesregierung (siehe sogleich).

${ }^{87}$ Vgl. ebd. 242.

${ }^{88}$ Art. 105 Abs. 1 B-VG i.d.F. § 26 Verfassungsnovelle 1925; positiv beurteilt von ADAMOVICH, Reform 240.

89 WITTMAYER, Verfassungs- und Verwaltungsreform 485.

${ }^{90}$ FrOEHLICH, Einleitung XIX; vgl. ADAMOVICH, Reform 228 (einer der "Hauptpunkte“).

${ }^{91} \S \S 8 f \quad$ V-ÜG 1920 i.d.F. §§ 3f. Übergangsnovelle BGBl. 1925/269.

92 Sog. „Ämter-der-Landesregierungen-Bundesverfassungsgesetz“ (BVG ÄmterLReg), BGBl. 1925/289. Der Wiener Magistrat fungierte schon seit 1922 als Amt der Landesregierung: ADAMOVICH, Reform 249.

${ }^{93} \S 5$ BVG ÄmterLReg.

${ }^{94}$ MERKL, Grundlagen 230. 
terstehenden Bezirkshauptmannschaften ${ }^{95}$ wurden nun 1925 eindeutig zu Behörden der Länder erklärt. ${ }^{66}$ Damit hatte man, wie Merkl beklagte, das „nächstliegende Mittel, um eine gewisse Einheitlichkeit der Bundesverwaltung $\mathrm{zu}$ sichern", nicht ergriffen, nämlich eine Ausdehnung der unmittelbaren Bundesverwaltung, ebensowenig die Möglichkeit, die Bezirkshauptmannschaften als „Fundament des Verwaltungsgebäudes" in Form von Bundesbehörden einzurichten. ${ }^{97}$

Die bis 1925 nebeneinander stehenden Apparate der früheren gesamtstaatlichen und der früheren autonomen Verwaltung wurden im Amt der Landesregierung vereinigt, das als neue "einheitliche Behörde" in organisatorischer Hinsicht Landesbehörde war. $^{98}$ Zeitgenossen charakterisierten die Veränderungen von 1925 daher mit den Schlagworten der "Verschmelzung“ der beiden „Amtsapparate“ unter „Beseitigung des [...] Doppelgeleises" sowie der „Verländerung" bisheriger Gesamtstaats- bzw. Bundesbehörden. ${ }^{99}$ Kritiker sahen darin eine „Auslieferung der Verwaltung an die Länder". ${ }^{100}$ Funktionell konnte das Amt der Landesregierung aber je nach betroffener Kompetenz entweder als Landes- oder als Bundesbehörde tätig werden. ${ }^{101}$ Damit war eine Möglichkeit geschaffen, die Verwaltung nach sachlichen Gesichtspunkten zu organisieren, also unabhängig von der kompetenzrechtlichen Einordnung. ${ }^{102}$ Dies

\footnotetext{
95 AdAMOVICH, Frage 49; DeRs., Reform 252.

${ }^{96} \S 8$ Abs. 4 V-ÜG i.d.F. § 3 ÜG-Novelle 1925; ADAMOVICH, Reform 252; WITTMAYER, Verfassungs- und Verwaltungsreform $484 \mathrm{f}$.

${ }^{97}$ MerKL, Epilog 1269.

${ }^{98}$ Dabei erschienen die Bundesbeamten „zum Aussterben bestimmt": WITTMAYER, Verfassungsentwicklung 461.

${ }^{99}$ ADAMOVICH, Reform 252, 243f. Kritisch zum Begriff „Verländerung“ KELSEN, Reform 28.

${ }^{100}$ STOLPER, Bund 733f.; ähnlich AUSTERLITZ, Verfassungsrevision 161ff.; STOLPER, Verfassungsreform 986 („Balkanisierung“).

101 ADAmOvich, Reform 250; Kelsen, Vollendung 311.

102 ADAMOVICH, Reform 250.
}

konnte gegenüber den ausländischen Geldgebern als wichtige Sparmaßnahme ausgegeben werden ${ }^{103}$ - man hätte darin aber auch ein Eingeständnis nicht völlig geglückter Kompetenzverteilung sehen können. Soweit erkennbar, wurde dieser Aspekt selbst von Kritikern der B-VG-Novelle nicht thematisiert.

\section{Themen}

Der Transformationsprozess von der Doppelgleisigkeit der Verwaltung zur mittelbaren Bundesverwaltung war also durch schrittweises Vorgehen, durch Provisorien, Experimente und die Erwartung weiterer Veränderungen ${ }^{104}$ gekennzeichnet. Daher wurden viele Fragen immer wieder neu thematisiert; die wissenschaftliche Auseinandersetzung blieb an den gesetzlichen Zäsuren nicht stehen und erstreckte sich oft über mehrere der oben definierten Phasen. Der beschränkte Umfang dieses Beitrags macht hier nur einen sehr skizzenhaften Überblick möglich.

Unterschiedliche Positionen gab es schon auf fundamental-begrifflicher Ebene. Ob die Novelle 1925 „Vollendung“105 oder „Reform" des B-VG und ob sie überhaupt eine „echte“ Reform im Sinne einer Verbesserung ${ }^{106}$ war, erschien ebenso strittig wie ihre Notwendigkeit. ${ }^{107}$ Auch die Sinnhaftigkeit einer bundesstaatlichen Verfassung für den national fast einheitlichen Klein-

\footnotetext{
${ }^{103}$ Vgl. WITTMAYER, Verfassungsentwicklung 461: „Sehr geschickt wurden die Genfer Abmachungen verwendet, um in der großen Novelle von 1925 verschiedene föderalistische Rechtsforderungen durchzusetzen."

104 ADAMOVICH, Reform 249.

${ }^{105}$ KELSEN, Vollendung; DERS., Reform.

${ }^{106}$ MANNLICHER, Verwaltungsreform 357f.

${ }^{107}$ AUSTERLITZ, Verfassungsrevision 162.
} 
staat, ein "Zwerggebilde", 108 wurde noch heftig angezweifelt. ${ }^{109}$

Umstritten war auch der stets wieder begegnende Begriff der „Doppelgleisigkeit“ (zeitgenössisch übrigens oft als „Doppelgeleise“110 bezeichnet), der vielfach als tendenziös empfunden wurde: Adamovich kritisierte, es sei „dieses irreführende Schlagwort meist nur zu Unrecht verwendet" ${ }^{\prime 111}$ worden, weil die Aufgaben von verschiedenen Behörden mit genauer Kompetenzabgrenzung vollzogen wurden. Damit war „nicht eine doppelte Bearbeitung gleichartiger Geschäftsfälle, sondern nur ein Zusammenwirken beider Behörden gegeben", Voraussetzung für einen Ausgleich zwischen gesamtstaatlichen und Landesinteressen. „Die vielgeschmähte Doppelgeleisigkeit bedeutete somit [...] für den Bereich der Landesverwaltung die Erfüllung des Grundsatzes der Trennung der Wirtschaftsverwaltung von der Hoheitsverwaltung".112 Auch Pendl bestritt „eine zur Gänze oder doch zum überwiegenden Teile hypertrophe Doppelverwaltung". Eine „wirkliche Doppelverwaltung", bei der „wesensgleiche Verwaltungsakte auf einem und demselben Verwaltungsgebiete von zweierlei Behörden [...] gesetzt werden“, existierte nicht; es sei „also nicht alles wirklich Doppelverwaltung, was man Doppelverwaltung nennt." ${ }^{113}$

Die Übernahme der (zuvor von einem beamteten Landeschef geleiteten) staatlichen Verwaltung durch eine gewählte Landesregierung galt als wichtiger Schritt zur weiteren Demokratisierung $^{114}$ der Verwaltung, die für die Bezirksver-

\footnotetext{
108 STOLPER, Bund 622.

${ }^{109}$ AusterlitZ, Verfassungsrevision 162; KELSEN, Reform; DERS., Vollendung 302f.; STOLPER, Bund 621ff.

110 ADAMOVICH, Reform 248f.; Ders., Frage 50; FrOeHLICH, Einleitung XIX; KELSEN, Reform; DERS., Stellung 114; STOLPER, Verfassungsreform 985.

111 ADAMOVICH, Reform 245.

112 Ebd. 246.

${ }^{113}$ PendL, Frage 115.

${ }^{114}$ Vgl. JABLONER, Bundesminister 23f.
}

waltung erst in Planung war. ${ }^{115}$ Diese Demokratisierung geriet jedoch in ein Spannungsverhältnis zum Prinzip der Verantwortlichkeit für die Verwaltungsführung. Die Verlagerung der gesamtstaatlichen Verwaltungsaufgaben von der Landesregierung (1918-1920) zum Landeshauptmann (1920) erschien als Wende von einer eher theoretischen kollegialen $\mathrm{zu}$ einer effektiven monokratischen Verantwortlichkeit. ${ }^{116}$ Deren Justizialisierung durch eine „Individual- und Schuldhaftung"117 nach Art. 142 B-VG hielt Kelsen für einen Fortschritt im Vergleich zur klassischen Bundesexekution, die als „technisch gänzlich rückständige [...] Kollektiv- und Erfolgshaftung" Unschuldige träfe. ${ }^{118}$ Kritiker wie Adamovich $^{119}$ oder Wittmayer ${ }^{120}$ bemängelten hingegen die "Schwerfälligkeit und Kompliziertheit"121 der Konstruktion einer mehrstufigen Weisungskette und erwarteten - nicht zu Unrecht, wie sich zeigen sollte ${ }^{122}$ - mangelnde praktische Bedeutung dieser ,letzte[n] Verteidigungslinie [...], auf die der Bund sich zurückzieht" ${ }^{123}$ Zugleich stellte sich die Frage, inwieweit man angesichts der faktischen Machtstellung der Landeshauptleute überhaupt noch eine Verantwortlichkeit der Bundesminister annehmen dürfe. Damit schien indirekt das Parlament geschwächt ${ }^{124}$ womit man wieder bei der Frage einer demokratisch legitimierten und kontrollierten Verwaltung war.

$115 \mathrm{Vgl}$. TREBITSCH, Demokratie 53ff.

116 ADAMOVICH, Reform 241; FroEHLICH, Eigentümlichkeiten 87ff.

117 KELSEN, Bundesexekution 179.

${ }^{118}$ Ebd. 164f., 177f.; vgl. FROEHLICH, Eigentümlichkeiten 88f.

${ }^{119}$ ADAMOVICH, Reform 242f.

120 WITTMAYER, Verfassungsentwicklung 460.

${ }^{121}$ ADAMOVICH, Reform 242f („,umständlich, zeitraubend und daher wenig praktikabel“).

${ }^{122}$ NESCHWARA, Verfassungsgerichtshof; VAŠEK, Verfassungsgerichtshof.

${ }^{123}$ WITTMAYER, Verfassungs- und Verwaltungsreform 485.

${ }^{124}$ AusTERLITZ, Verfassungsrevision 163f. (Bundesregierung als „der reine Niemand“). 
In Verbindung mit der Verantwortlichkeit standen auch die Fragen, ob die Landesregierung nach Mehrheits- oder nach Verhältniswahlrecht ${ }^{125}$ gewählt und ob sie sodann nach Ministerial- oder Kollegialsystem organisiert sein sollte. ${ }^{126}$

Verantwortlichkeit ist die Kehrseite der Verwaltungsführung, deren Einheitlichkeit ebenfalls problematisiert wurde. In diesem Zusammenhang erschien die mittelbare Bundesverwaltung als Gefahr für eine gleichförmige Verwaltung, die sich manche nur unter zentraler Leitung durch die Bundesminister vorstellen konnten. Die Verwaltungsverfahrensgesetze wurden als eine Art Gegengewicht zur Übertragung von Verwaltungsaufgaben an die Länder gesehen ${ }^{127}$ - unterschiedliche Verfahrensregeln wären auch unvorstellbar gewesen. ${ }^{128}$ Die vereinheitlichende Kraft der Verwaltungsgerichtsbarkeit ${ }^{129}$ wurde angezweifelt. Auch hier stand das Modell der Justizialisierung in der Kritik - teils aus praktischen Gründen,130 teils aus ideologischen, weil eine spätere Machtübernahme durch die Arbeiterklasse von einem zentral gesteuerten Verwaltungsapparat erleichtert worden wäre. ${ }^{131}$ Andere plädierten schon Anfang der 1920er-Jahre für eine „von unten auf organisierte Verwaltungsrechtsprechung" als Gegengewicht zur stärkeren Politisierung der Verwaltung und damit für

\footnotetext{
${ }^{125}$ KLEIN, Verhältniswahl 97ff.; KoCH, Frage 56; PENDL, Frage 105.

${ }^{126}$ WITTMAYER, Verfassungsentwicklung 463; vgl. WIEDERIN, Ressortsystem.

${ }^{127}$ MANNLICHER, Verwaltungsreform 367.

128 Ebd. 358

129 ADAMOVICH, Reform 264ff.

${ }^{130}$ BARTSCH, Reform 31.

${ }^{131}$ AUsterlitZ, Verfassungsrevision 164, kritisierte dabei auch die „Verfassungsfachmänner[...], die hier eine günstige Gelegenheit wahrnehmen, ihre Rechtstheorien in die Tat umzusetzen und jene ideale Verfassung zu schaffen, in der alles, was Macht ist, ausgetilgt und durch Gerichte ersetzt werden soll“ - klarer Angriff auf Kelsen und weiterer Beleg für die schwierige Beziehung zwischen den beiden Verfassungsrichtern, vgl. OLECHOWSKI, Kelsen 431.
}

das (erst 2014 realisierte) Konzept einer mehrstufigen Verwaltungsgerichtsbarkeit. ${ }^{132}$

Schließlich wurde in allen Phasen des Übergangs auch das Schicksal der Staatsbeamten thematisiert. ${ }^{133}$ Hier begegneten einander tagespolitisch-strategische Überlegungen - die Beamten der staatlichen Verwaltung stellten eine beachtliche Wählergruppe, die Beamtenfrage war daher politisch interessant - mit grundsätzlichen Gedanken zur Bedeutung des traditionellen österreichischen Beamtentums und dessen Vorteilen im Vergleich zu gewählten Funktionären. ${ }^{134}$

\section{Conclusio}

Dieser Überblick hat gezeigt, dass die Verfassungsnovelle $1925 \mathrm{zu}$ Unrecht im Schatten der Verfassungsnovelle 1929 steht. Dies gilt sowohl von einem allgemeinen als auch von einem individuellen Standpunkt aus: Reduziert man 1929 auf die Aufwertung des Bundespräsidenten, so kann man 1925 auf die Festschreibung der Machtstellung für die Landeshauptleute zuspitzen. In der österreichischen „Realverfassung" spielen die Landeshauptleute, die eine politische Leitungsfunktion innerhalb des Landes mit einer „unpolitischen " Verwaltungsfunktion verbinden, ${ }^{135}$ eine kontinuierlich stärkere Rolle als der Bundespräsident. Der Umbau von der gewaltenverbindenden parlamentarischen Republik zur gewaltenteilenden parlamentarischen Präsidentschaftsrepublik mag zwar spektakulärer gewesen sein, im Alltag begegnen Ämter der Landesregierung und mittel-

\footnotetext{
${ }^{132} \mathrm{KoCH}$, Frage 59f.

133 ADAmOvich, Reform 252f.; Kelsen, Reform 29; DERS., Vollendung 307, 314; MERKL, Grundlagen 224; PendL, Frage 109ff.; STOLPER, Bund 733f; DeRS., Verfassungsreform 985 (vgl. KoHL, Gesetzgebung); WiTTMAYER, Verfassungsentwicklung 462; DERS., Verfassungs- und Verwaltungsreform 48.

${ }^{134}$ MERKL, Epilog 1270.

135 PURTSCHER, Organisationshoheit 338f.
} 
bare Bundesverwaltung den Staatsbürgern jedoch erheblich häufiger.

\section{Korrespondenz:}

Prof. Dr. Gerald KOHL

Universität Wien

Institut für Rechts- und Verfassungsgeschichte

Schottenbastei 10-16

1010 Wien

gerald.kohl@univie.ac.at

ORCID-Nr. 0000-0002-6812-3897

\section{Abkürzungen:}

LReg. Landesregierung

V-ÜG Verfassungs-Übergangsgesetz

Siehe auch das allgemeine Abkürzungsverzeichnis: [http://www.rechtsgeschichte.at/files/abk.pdf]

\section{Literatur:}

Ludwig Adamovich, Georg Froehlich (Hgg.), Die Novellen zur Bundesverfassung (Wien 1926).

Ludwig ADAMOVICH, Die österreichische Verfassungsund Verwaltungsreform, in: Juristische Wochenschrift 55 (1926) 1901-1903.

DERS., Die Reform der österreichischen Bundesverfassung, in: Zeitschrift für öffentliches Recht 5 (1926) 228-280.

DERS., Die Typen der Kompetenzverteilung zwischen Bund und Ländern nach der Bundes-Verfassungsnovelle von 1929, in: Österreichisches Verwaltungsblatt 1/3 (1930) 3, 7-9.

DERS., Zur Frage der verfassungsmäßigen Organisation der Landesverwaltung in Österreich, in: Zeitschrift für Verwaltung 56 (1923) 33-62.

Arbeitsgemeinschaft Österreichische Rechtsgeschichte (Hg.), Manual Rechts- und Verfassungsgeschichte (Wien 52018).

Friedrich AUSTERLITZ, Verfassungsrevision, in: Der Kampf 18/5 (1925) 161-165.

Robert BARTSCH, Die Reform der Bundesverfassung. Bericht über die Wechselrede in der Wiener Juristischen Gesellschaft am 9. Dezember 1925, in: Gerichts-Zeitung 77 (1926) 30-31.

Wilhelm BRAUNEDER, Deutschösterreich 1918. Die Republik entsteht (Wien 2000).
DERS., Geschichte der Struktur der allgemeinen Verwaltung, in: Bundeskanzleramt (Hg.), Die öffentliche Verwaltung in Österreich (Wien 1992) 31-59.

DERS., Österreichische Verfassungsgeschichte (Wien 112009).

Peter BUßJÄGER, Die rechtliche und politische Kontrolle der Länder und ihrer Organe bei der Vollziehung von Bundesrecht im System der österreichischen Bundesverfassung, in: Peter BUßJÄGER (Hg.), Vollzug von Bundesrecht durch die Länder (= Schriftenreihe des Instituts für Föderalismus 85, Wien 2002) 5-22.

Georg FROEHLICH, Der Landeshauptmann als Organ der Bundesverwaltung, in: Zeitschrift für Verwaltung 54 (1921) 131-135.

Ders., Einleitung, in: Ludwig ADAMOvich, Georg FrOEHLICH (Hgg.), Die Novellen zur Bundesverfassung (Wien 1926) XIII-XXXIX.

DERS., Staatsrechtliche Eigentümlichkeiten der österreichischen Bundesverfassung, in: Zeitschrift für Verwaltung 54 (1921) 81-90.

Anna GAMPER, Zur verfassungsrechtlichen Systemrelevanz der Bezirksverwaltungsbehörden in der mittelbaren Bundesverwaltung, in: Peter BUßJ̈̈GER u.a. (Hg.), Kontinuität und Wandel. Von ,guter Polizey" zum Bürgerservice. Festschrift 150 Jahre Bezirkshauptmannschaften (= Institut für Föderalismus - Schriftenreihe 125, Wien 2018) 555-575.

Ernst C. Hellbling, Die Landesverwaltung in Cisleithanien, in: Adam WANDRUSZKA, Peter URBANITSCH (Hgg.), Die Habsburgermonarchie 1848-1918, Bd. 2: Verwaltung und Rechtswesen (Wien 1975) 190-269.

Rudolf HERRNRITT, Österreichisches Verwaltungsrecht. Ein Grundriß der Rechtstheorie und Gesetzgebung der inneren Verwaltung (Tübingen 1925).

Michael HöLlBACHER, Die Kehrseite der Medaille Ausgewählte Problemfelder des Vollzugszentralismus, in: Melanie GIERLINGER u.a. (Hg.), Subsidiarität, Föderalismus, Selbstverwaltung (Wien 2012) 81-106.

Gottfried Holzer, Weinaufsicht und bundesstaatliches Prinzip. Bemerkungen zum Erkenntnis des Verfassungsgerichtshofes vom 1. Juli 1987, G 78/87-9, in: Zeitschrift für Verwaltung (1988) 126130.

Gerhart Holzinger, Aktuelle Fragen des Bundesstaatsprinzips. Zum Stand der Bundesstaatsreform Überlegungen im Österreich Konvent, in: Walter BERKA u.a. (Hgg.), Verfassungsreform. Überlegungen zur Arbeit des Österreich-Konvents (Wien-Graz 2004) 71-83. 
Clemens JABLONER, Bundesminister und mittelbare Bundesverwaltung, in: DERS., Methodenreinheit und Erkenntnisvielfalt. Aufsätze zur Rechtstheorie, Rechtsdogmatik und Rechtsgeschichte (=Schriftenreihe des Hans Kelsen-Instituts 25, Wien 2013) 23-46.

Hans KELSEN, Die Bundesexekution. Ein Beitrag zur Theorie und Praxis des Bundessstaates, unter besonderer Berücksichtigung der Deutschen Reichsund der österreichischen Bundes-Verfassung (Tübingen 1927).

DERS., Die Organisation der vollziehenden Gewalt, in: Zeitschrift für öffentliches Recht 1 (1919/1920) 48-60.

DERS., Die Reform der Bundesverfassung. Bericht über die Wechselrede in der Wiener Juristischen Gesellschaft am 9. Dezember 1925, in: GerichtsZeitung 77 (1926) 28-30.

DERS., Die Stellung der Länder in der künftigen Verfassung Deutschösterreichs, in: Zeitschrift für öffentliches Recht 1 (1919/1920) 98-122.

DERS., Die Vollendung der österreichischen Bundesverfassung, in: Zeitschrift für Politik 15 (1926) 301-319.

Franz KLEIN, Die Verhältniswahl der Landesregierung, in: Der Österreichische Volkswirt 19/4 (1926) 97-99.

Hans $\mathrm{KOCH}$, Zur Frage der verfassungsmäßigen Organisation der Landesverwaltung in Österreich, in: Zeitschrift für Verwaltung 56 (1923) 56-61.

Gerald KOHL, Das bundesstaatliche Prinzip in der österreichischen Verfassungsrechtswissenschaft der Ersten Republik, in: Martin P. SCHENNACH (Hg.), Rechtshistorische Aspekte des österreichischen Föderalismus (Wien 2015) 117-145.

DERS., Die außerordentliche Gesetzgebung im Rahmen der Genfer Protokolle. Theorie und Praxis des „außerordentlichen Kabinettsrates“ 1922-1924, in: Christian Neschwara u.a. (Hgg.), Normsetzung im Notstand. Außerordentliche Gesetzgebungsbefugnisse im 19. und 20. Jahrhundert (=BRGÖ 2018/2, Wien 2018) 318-343.

Egbert MANNLICHER, Die österreichische Verwaltungsreform des Jahres 1925, in: Zeitschrift für öffentliches Recht 5 (1926) 357-394.

Adolf MerkL, Demokratie und Reform der Verwaltung, in: Zeitschrift für öffentliches Recht 2 (1921) 65-137.

DERS., Die verfassungsrechtlichen Grundlagen der österreichischen Verwaltung, in: Zeitschrift für öffentliches Recht 2 (1921) 208-239.

DERS., Epilog zur Verfassungsreform, in: Der Österreichische Volkswirt 17 (1925) 1241-1243, 12691272.
DERS., Erläuterungen zum Gesetz vom 14. November 1918 betreffend die Übernahme der Staatsgewalt in den Ländern, StGBl Nr. 24, in: Hans Kelsen, Die Verfassungsgesetze der Republik Deutschösterreich Bd. 1 (Wien-Leipzig 1919) 103-114.

DERS., Länderautonomie, in: Österreichische Zeitschrift für Verwaltung 50 (1917) 69-71.

DERS., Staat und Länder, in: Zeitschrift für Verwaltung 53/1 (1920) 1-2.

DERS., Zum Problem der Verwaltungsreform, in: Zeitschrift für öffentliches Recht 2 (1921) 163-174.

DERS., Zum rechtstechnischen Problem der bundesstaatlichen Kompetenzverteilung. Mit besonderer Berücksichtigung der deutschösterreichischen Bundesverfassung, in: Zeitschrift für öffentliches Recht 2 (1921) 336-359.

Christian NESCHWARA, Der österreichische Verfassungsgerichtshof als Staatsgerichtshof der Republik Österreich von 1920 bis 1934, in: Gerald KOHL, István SzABÓ (Hgg.), Staatsgerichtsbarkeit in Mitteleuropa. Beiträge zur Tagung „Staatsgerichtsbarkeit in Vergangenheit und Gegenwart" (Budapest 2017) 59-68.

Julius OFNER, Die neue Bundesverfassung, in: Juristische Blätter 50 (1921) 65-70.

Thomas OLECHOWSKI, Hans Kelsen. Biographie eines Rechtswissenschaftlers (Tübingen 2020).

Wilhelm PENDL, Zur Frage der verfassungsmäßigen Organisation der Landesverwaltung in Österreich, in: Zeitschrift für Verwaltung 56 (1923) 104-121.

Wolfgang PESENDORfER, Der Landeshauptmann. Historische Entwicklung, Wesen und verfassungsrechtliche Gestalt einer Institution (= Forschungen aus Staat und Recht 76, Wien-New York 1986).

Markus PURTSCHER, Die Organisationshoheit und der Behördenbegriff in der mittelbaren Bundesverwaltung, in: Juristische Blätter 102 (1980) 338-348.

Christian RANACHER, Die allgemeine staatliche Verwaltung in den Ländern als effiziente Regierungsorganisation - Plädoyer für ein nach wie vor modernes Konzept, in: Journal für Rechtspolitik 23 (2015) 199-208.

Willibald Rosner, Reinelde Motz-LinHART (Hgg.), 1861 und die Folgen. Region und Parlamentarismus. Die Vorträge des NÖ Instituts für Landeskunde, Wien, Palais Niederösterreich, 5. bis 6. Juli 2011 (=Studien und Forschungen aus dem Niederösterreichischen Institut für Landeskunde 56, St. Pölten 2013).

Georg SCHMITZ, Der Landesamtsdirektor. Entstehung und Entwicklung (Wien 1977).

Wolfgang STEINER, Landeshauptmann, in: Erich PüRGY (Hg.), Das Recht der Länder - System, Bd. 1: 
Landesverfassungsrecht und Organisationsrecht (Wien 2012) 385-421.

Gustav STOLPER, Bund und Länder, in: Der österreichische Volkswirt 17 (1925) 593-595, 621-623, 705706, 733-734.

DERS., Verfassungsreform, in: Der österreichische Volkswirt 17 (1925) 985-987.

Oskar TREBITSCH, Demokratie und Verwaltung, in: Der Kampf 18/2 (1925) 53-58.

Josef ULBRICH, Das österreichische Staatsrecht (= Das öffentliche Recht der Gegenwart 10, Tübingen 1909).

Markus VAŠEK, Der österreichische Verfassungsgerichtshof als Staatsgerichtshof nach 1945, in: Gerald KOHL, István SzABó (Hgg.), Staatsgerichtsbarkeit in Mitteleuropa. Beiträge zur Tagung "Staatsgerichtsbarkeit in Vergangenheit und Gegenwart" (Budapest 2017) 163-177.

Vollversammlung der Wiener Juristischen Gesellschaft am 7. Februar 1917, in: Juristische Blätter 46 (1917) 76-78.
Karl WeBer, Die mittelbare Bundesverwaltung. Eine verfassungs- und verwaltungsrechtliche Untersuchung der Organisation der Verwaltung des Bundes im Bereich der Länder außer Wien. Zugleich eine Geschichte der Behörden der allgemeinen staatlichen Verwaltung in den Ländern außer Wien (Wien 1987).

Ewald WIEDERIN, Das Ressortsystem in der Landesregierung, in: Zeitschrift für öffentliches Recht 73 (2018) 507-524.

DERS., Die Diskussion über die Stellung der Länder in der Zeitschrift für öffentliches Recht, in: Zeitschrift für öffentliches Recht 69 (2014) 875-891.

Leo WITTMAYER, Die österreichische Verfassungs- und Verwaltungsreform, in: Deutsche Juristen-Zeitung 31 (1926) 484-487.

DERS., Österreichs Verfassungsentwicklung. Ein Beitrag zur Anschlußfrage, in: Zeitschrift für die gesamte Staatswissenschaft 83/3 (1927) 449-473. 